\title{
ISSPIC 14 Organisation
}

International Advisory Committee

ALONSO J.A. - University of Valladolid, Spain

BONAČIĆ-KOUTECKÝ V. - Humbolt-University, Berlin, Germany

BRÉCHIGNAC C. - CNRS, Orsay, France

BROYER M. - University Claude Bernard, Lyon, France

CAMPBELL E.E.B. - Göteborg University, Sweden

CASTlEMAN A.W. - Pennsylvania State University, University Park, USA

CHEKMAREV S.F. - Institute of Thermophysics, Novosibirsk, Russia

CHESHNOVSKY O. - Tel Aviv University, Israel

HABERLAND H. - University of Freiburg, Germany

HARUTA M. - Tokyo Metropolitan University, Japan

HENRY C. - University of Marseille, Marseille, France

JARROLD M.F. - Indiana University, Bloomington, USA

KAPPES M. - University of Karlsruhe, Germany

KAYA K. - RIKEN, Saitama, Japan

KONDOW T. - Toyota Technological Institute, Chiba, Japan

LANDMAN U. - Georgia Institute of Technology, Atlanta, USA

LIEVENS P. - Catholic University Leuven, Belgium

MÄRK T.D. - Leopold Franzen University, Innsbruck, Austria

MANNINEN M. - University of Jyväskylä, Finland

MILANI P. - University of Milano, Italy

PALMER R.E. - University of Birmingham, UK

VALLÉE F. - University of Bordeaux 1, France

WANG G.H. - Nanjing University, China

WANG L.S. - Washington State University, Richland, USA

WÖSTE L. - Freie University Berlin, Germany 


\section{Emeritus Members}

BERRY R.S. - University of Chicago, USA

BUTTET J. - École Polytechnique Fédérale, Lausanne, Switzerland

FRIEDEL J. - University Paris-Sud, France

GILLET M.F. - University Paul Cezanne, Aix Marseille, France

HENSEL F. - Philips University, Marburg, Germany

JORTNER J. - Tel Aviv University, Israel

MARTIN T.P. - Max Planck Institute, Stuttgart, Germany

MONOT R. - École Polytechnique Fédérale, Lausanne, Switzerland

MÜHLSCHLEGEL B. - Köln University, Germany (deceased)

ROSEN A. - Göteborg University, Sweden

SUGANO S. - University of Tokyo, Japan

\section{Organising Committee}

\section{Chairpersons:}

Julio A. ALONSO - University of Valladolid, Spain

Luis C. BALBÁS - University of Valladolid, Spain

Conference secretary:

María J. LÓPEZ - University of Valladolid, Spain

\section{Members:}

Andrés AGUADO - University of Valladolid, Spain

Iván CABRIA - University of Valladolid, Spain

Nicolás A. CORDERO - University of Burgos, Spain

Ángel GONZÁLEZ UREÑA - Universidad Complutense, Madrid, Spain

Ángel MAÑANES - University of Cantabria, Santander, Spain

Fernando MARTÍN - Autonoma University of Madrid, Spain

Luis M. MOLINA - University of Valladolid, Spain

Josep M. OLIVA - Chemical Physics Institute, CSIC, Madrid, Spain

Begoña TORRES - University of Burgos, Spain

\section{Information about the Guest Editors of the ISSPIC 14 Proceedings}

Andrés AGUADO

Departamento de Física Teórica, Atómica y Óptica, Universidad de Valladolid, 47011 Valladolid, Spain

Luís M. MOLINA

Departamento de Física Teórica, Atómica y Óptica, Universidad de Valladolid, 47011 Valladolid, Spain

\section{Ángel MAÑANES}

Departamento de Fsica Moderna, Universidad de Cantabria, 37005 Santander, Spain

Begoña TORRES

Departamento de Matemáticas y Computación, Universidad de Burgos, 09006 Burgos, Spain 